\title{
Double C-H Activation Strategy for the Asymmetric Synthesis of $C_{2}$ Symmetric Anilines
}

\author{
Huw M. L. Davies* and Qihui Jin \\ Department of Chemistry, University at Buffalo, State University of New York, Buffalo, \\ New York 14260-3000, USA
}

Supporting Information

A: Experimental Procedures (S1-S13)

B: Selected Spectra (S14-S16) 


\section{Experimental Section:}

General Procedure: A: To a stirring solution of $N, N$-dimethylanilines $(1 \mathrm{mmol})$ and $\mathrm{Rh}_{2}(S \text {-DOSP })_{4}(0.005 \mathrm{mmol})$ in 2,2-dimethylbutane $(2 \mathrm{~mL})$ was added a degassed solution of methyl $p$-bromophenyldiazoacetate $4(0.5 \mathrm{mmol})$ in 2,2-dimethylbutane (5 $\mathrm{mL}$ ) over $45 \mathrm{~min}$ via a syringe-pump at room temperature. The resulting mixture was allowed to stir for another $30 \mathrm{~min}$. The solvent was evaporated under reduced pressure and the residue was subjected to flash chromatography on silica gel.

B: To a stirring solution of $N, N$-dimethylanilines $(0.5 \mathrm{mmol})$ and $\mathrm{Rh}_{2}(S \text {-DOSP })_{4}(0.01$ mmol) in 2,2-dimethylbutane $(2 \mathrm{~mL})$ was added a degassed solution of methyl $p$ bromophenyldiazoacetate $4(1.0 \sim 1.5 \mathrm{mmol})$ in 2,2-dimethylbutane $(8 \mathrm{~mL})$ in $45 \mathrm{~min}$ via a syringe-pump at room temperature. The resulting mixture was allowed to stir for another $30 \mathrm{~min}$. The solvent was evaporated under reduced pressure and the residue was subjected to flash chromatography on silica gel.

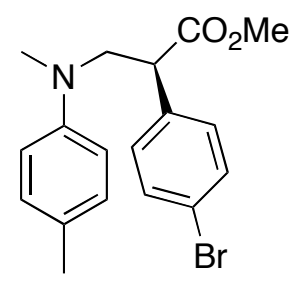

7a: Procedure A, pale yellow oil: $\mathrm{R}_{\mathrm{f}} 0.33$ (5:1 pentane/ether); [a] ${ }_{\mathrm{D}}^{25} \square 86.3$ (c $1.85, \mathrm{CHCl}_{3}$ ); FTIR (film) 3010, 2949, 2917, 1735, 1618, 1521, 1488, 1199, 1164, 1011, $803 \mathrm{~cm}^{-1} ;{ }^{1} \mathrm{H}$ NMR (500 MHz, $\left.\mathrm{CDCl}_{3}\right) \square 7.44(\mathrm{~d}, J=8.5 \mathrm{~Hz}, 2 \mathrm{H}), 7.19(\mathrm{~d}, J=8.5 \mathrm{~Hz}, 2 \mathrm{H}), 7.05(\mathrm{~d}, J=$ $8.5 \mathrm{~Hz}, 2 \mathrm{H}), 6.60$ (d, $J=8.5 \mathrm{~Hz}, 2 \mathrm{H}), 4.04$ (dd, $J=14.3,8.2 \mathrm{~Hz}, 1 \mathrm{H}), 3.97$ (dd, $J=8.2$, $5.6 \mathrm{~Hz}, 1 \mathrm{H}), 3.63(\mathrm{~s}, 3 \mathrm{H}), 3.52(\mathrm{dd}, J=14.3,5.6 \mathrm{~Hz}, 1 \mathrm{H}), 2.77(\mathrm{~s}, 3 \mathrm{H}), 2.25(\mathrm{~s}, 3 \mathrm{H}) ;{ }^{13} \mathrm{C}$ NMR (125 MHz, $\left.\mathrm{CDCl}_{3}\right) \square$ 173.1, 146.2, 136.1, 131.9, 129.8 (2C), 126.1, 121.6, 112.6, 56.9, 52.2, 49.0, 39.4, 20.2; LC-MS (ESI) $\mathrm{m} / z$ (relative intensity) 134 (100), 201 (5), 362 $\left([\mathrm{M}+\mathrm{H}]^{+}, 5\right)$; HPLC analysis: $53 \%$ ee (Chiralcel OJ, $10.0 \% i$-PrOH in hexane, 1.0 $\mathrm{mL} / \mathrm{min}, \square=254 \mathrm{~nm}, \mathrm{t}_{\mathrm{R}}=23.9 \mathrm{~min}$, minor; $\mathrm{t}_{\mathrm{R}}=35.6$ min, major). Anal. Calcd for $\mathrm{C}_{18} \mathrm{H}_{20} \mathrm{BrNO}_{2}$ : C, 59.68; H, 5.56; N, 3.87. Found: C, 59.73; H, 5.61; N, 3.83. 


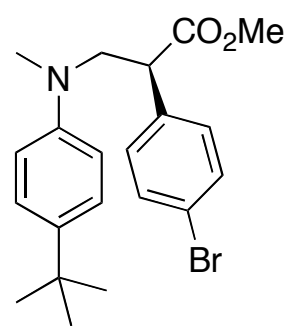

7b: Procedure A: colorless oil; $\mathrm{R}_{\mathrm{f}} 0.56$ (5:1 pentane/ether); [a] ${ }_{\mathrm{D}}^{25} \square 85.3$ (c 5.50, $\mathrm{CHCl}_{3}$ ); FTIR (film) 2953, 2901, 2866, 1736, 1613, 1520, 1488, 1199, 1164, 1011, $815 \mathrm{~cm}^{-1} ;{ }^{1} \mathrm{H}$ NMR (500 MHz, $\left.\mathrm{CDCl}_{3}\right) \square 7.45(\mathrm{~d}, J=8.4 \mathrm{~Hz}, 2 \mathrm{H}), 7.27(\mathrm{~d}, J=8.8 \mathrm{~Hz}, 2 \mathrm{H}), 7.21(\mathrm{~d}, J=$ $8.4 \mathrm{~Hz}, 2 \mathrm{H}), 6.64(\mathrm{~d}, J=8.8 \mathrm{~Hz}, 2 \mathrm{H}), 4.04(\mathrm{dd}, J=14.3,8.5 \mathrm{~Hz}, 1 \mathrm{H}), 3.98(\mathrm{dd}, J=8.5$, $5.3 \mathrm{~Hz}, 1 \mathrm{H}), 3.63(\mathrm{~s}, 3 \mathrm{H}), 3.53(\mathrm{dd}, J=14.3,5.3 \mathrm{~Hz}, 1 \mathrm{H}), 2.80(\mathrm{~s}, 3 \mathrm{H}), 1.29(\mathrm{~s}, 9 \mathrm{H}) ;{ }^{13} \mathrm{C}$ NMR (125 MHz, $\left.\mathrm{CDCl}_{3}\right) \square 173.1,146.1,139.4,136.1,131.8,129.7,126.0,121.6,112.0$, 56.9, 52.1, 49.1, 39.2, 33.7, 31.5; LC-MS (ESI) $\mathrm{m} / z$ (relative intensity) 176 (100), 404 $\left([\mathrm{M}+\mathrm{H}]^{+}, 36\right)$; HPLC analysis: $58 \%$ ee (Chiralpak AD-RH, 0.5\% $i$-PrOH in hexane, 1.0 $\mathrm{mL} / \mathrm{min}, \quad \square=254 \mathrm{~nm}, \mathrm{t}_{\mathrm{R}}=6.2 \mathrm{~min}$, major; $\mathrm{t}_{\mathrm{R}}=7.2 \mathrm{~min}$, minor). Anal. Calcd for $\mathrm{C}_{21} \mathrm{H}_{26} \mathrm{BrNO}_{2}$ : C, 62.38; H, 6.48; N, 3.46. Found: C, 62.09; H, 6.52; N, 3.41.

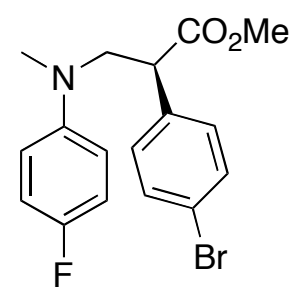

7c: Procedure A: pale yellow oil: $\mathrm{R}_{\mathrm{f}} 0.40$ (5:1 pentane/ether); [a] ${ }_{\mathrm{D}}^{25} \square 66.5$ (c 4.60, $\mathrm{CHCl}_{3}$ ); FTIR (film) 2951, 2917, 1735, 1512, 1488, 1230, 1201, 1164, $1011 \mathrm{~cm}^{-1} ;{ }^{1} \mathrm{H}$ NMR (500 $\left.\mathrm{MHz}, \mathrm{CDCl}_{3}\right) \square 7.50(\mathrm{~d}, J=8.5 \mathrm{~Hz}, 2 \mathrm{H}), 7.23(\mathrm{~d}, J=8.5 \mathrm{~Hz}, 2 \mathrm{H}), 7.00(\mathrm{dd}, J=9.2,8.2$ $\mathrm{Hz}, 2 \mathrm{H}), 6.66(\mathrm{dd}, J=9.2,4.3 \mathrm{~Hz}, 2 \mathrm{H}), 4.08(\mathrm{dd}, J=14.4,8.5 \mathrm{~Hz}, 1 \mathrm{H}), 4.00(\mathrm{dd}, J=8.5$, $5.8 \mathrm{~Hz}, 1 \mathrm{H}), 3.69$ (s, 3H), 3.54 (dd, $J=14.4,5.8 \mathrm{~Hz}, 1 \mathrm{H}), 2.81(\mathrm{~s}, 3 \mathrm{H}) ;{ }^{13} \mathrm{C}$ NMR $(125$ $\left.\mathrm{MHz}, \mathrm{CDCl}_{3}\right) \square 172.9,155.6(\mathrm{~d}, J=235.7 \mathrm{~Hz}), 145.1(\mathrm{~d}, J=1.7 \mathrm{~Hz}), 135.8,131.9,129.7$, 121.6, $115.6(\mathrm{~d}, J=22.0 \mathrm{~Hz}), 113.6(\mathrm{~d}, J=7.4 \mathrm{~Hz}), 57.3,52.2,48.9$, 39.6; LC-MS (ESI) $\mathrm{m} / \mathrm{z}$ (relative intensity) $366\left([\mathrm{M}+\mathrm{H}]^{+}, 100\right)$; HPLC analysis: $58 \%$ ee (Chiralcel OJ, $10.0 \%$ $i$-PrOH in hexane, $0.8 \mathrm{~mL} / \mathrm{min}, \square=254 \mathrm{~nm}, \mathrm{t}_{\mathrm{R}}=35.8 \mathrm{~min}$, major; $\mathrm{t}_{\mathrm{R}}=40.4 \mathrm{~min}$, minor). Anal. Calcd for $\mathrm{C}_{17} \mathrm{H}_{17} \mathrm{BrFNO}_{2}$ : C, 55.75; H, 4.68; N, 3.82. Found: C, 55.95; H, 4.73; N, 3.66 . 


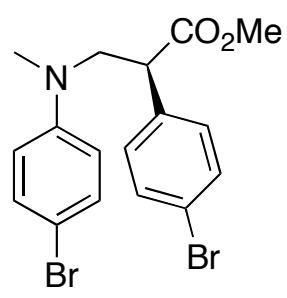

7d: Procedure A: colorless oil: $\mathrm{R}_{\mathrm{f}} 0.38$ (5:1 pentane/ether); [a] ${ }_{\mathrm{D}}^{25} \square 86.3$ (c 5.90, $\mathrm{CHCl}_{3}$ ); FTIR (film) 2949, 1735, 1591, 1497, 1199, $1165 \mathrm{~cm}^{-1}$; ${ }^{1} \mathrm{H}$ NMR (400 MHz, $\mathrm{CDCl}_{3}$ ) $7.44(\mathrm{~d}, J=8.4 \mathrm{~Hz}, 2 \mathrm{H}), 7.28(\mathrm{~d}, J=9.0 \mathrm{~Hz}, 2 \mathrm{H}), 7.16(\mathrm{~d}, J=8.4 \mathrm{~Hz}, 2 \mathrm{H}), 6.52(\mathrm{~d}, J=$ $9.0 \mathrm{~Hz}, 2 \mathrm{H}), 4.05$ (dd, $J=14.6,8.0 \mathrm{~Hz}, 1 \mathrm{H}), 3.93(\mathrm{dd}, J=8.0,5.8 \mathrm{~Hz}, 1 \mathrm{H}), 3.64(\mathrm{~s}, 3 \mathrm{H})$, $3.52(\mathrm{dd}, J=14.6,5.8 \mathrm{~Hz}, 1 \mathrm{H}), 2.75(\mathrm{~s}, 3 \mathrm{H}) ;{ }^{13} \mathrm{C} \mathrm{NMR}\left(75 \mathrm{MHz}, \mathrm{CDCl}_{3}\right) \square 172.7,147.1$, 135.7, 131.93, 131.88, 129.7, 121.7, 113.8, 108.8, 56.4, 52.2, 48.7, 39.3; LC-MS (ESI) $\mathrm{m} / \mathrm{z}$ (relative intensity) $426\left([\mathrm{M}+\mathrm{H}]^{+}, 49\right)$; HPLC analysis: $56 \%$ ee (Chiralcel OJ, $50.0 \% \mathrm{i}$ PrOH in hexane, $1.0 \mathrm{~mL} / \mathrm{min}, \square=254 \mathrm{~nm}, \mathrm{t}_{\mathrm{R}}=20.8 \mathrm{~min}$, major; $\mathrm{t}_{\mathrm{R}}=33.7$ min, minor). Anal. Calcd for $\mathrm{C}_{17} \mathrm{H}_{17} \mathrm{Br}_{2} \mathrm{NO}_{2}$ : C, 47.80; H, 4.01; N, 3.28. Found: C, 47.67; H, 3.96; N, 3.26 .

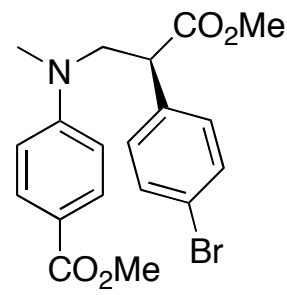

7e: Procedure A: white solid: mp 119-120 ${ }^{\circ} \mathrm{C} ; \mathrm{R}_{\mathrm{f}} 0.28$ (2:1 pentane/ether); [a] ${ }_{\mathrm{D}}^{25} \square 130.0$ ( $c$ 4.50, $\left.\mathrm{CHCl}_{3}\right)$; FTIR $\left(\mathrm{CHCl}_{3}\right)$ 2949, 1735, 1706, 1607, 1523, 1434, 1284, 1186, $1112 \mathrm{~cm}^{-1}$; ${ }^{1} \mathrm{H}$ NMR (500 MHz, $\left.\mathrm{CDCl}_{3}\right) \square 7.92(\mathrm{~d}, J=9.1 \mathrm{~Hz}, 2 \mathrm{H}), 7.47(\mathrm{~d}, J=8.4 \mathrm{~Hz}, 2 \mathrm{H}), 7.18(\mathrm{~d}$, $J=8.4 \mathrm{~Hz}, 2 \mathrm{H}), 6.62(\mathrm{~d}, J=9.1 \mathrm{~Hz}, 2 \mathrm{H}), 4.15(\mathrm{dd}, J=14.7,7.8 \mathrm{~Hz}, 1 \mathrm{H}), 3.98(\mathrm{dd}, J=$ 7.8, $6.4 \mathrm{~Hz}, 1 \mathrm{H}), 3.86(\mathrm{~s}, 3 \mathrm{H}), 3.66$ (s, 3H), $3.64(\mathrm{dd}, J=14.7,6.4 \mathrm{~Hz}, 1 \mathrm{H}), 2.84(\mathrm{~s}, 3 \mathrm{H})$; ${ }^{13} \mathrm{C}$ NMR $\left(125 \mathrm{MHz}, \mathrm{CDCl}_{3}\right) \square 172.6,167.2,151.2,135.4,132.0,131.4,129.7,121.9$, 117.7, 110.8, 55.9, 52.3, 51.5, 48.6, 39.5; LC-MS (ESI) $\mathrm{m} / z$ (relative intensity) 178 (50), $406\left([\mathrm{M}+\mathrm{H}]^{+}, 27\right), 428\left([\mathrm{M}+\mathrm{Na}]^{+}, 96\right)$; HPLC analysis: $71 \%$ ee (Chiralpak AD-RH, $20.0 \% i$-PrOH in hexane, $1.0 \mathrm{~mL} / \mathrm{min}, \mathrm{C}=254 \mathrm{~nm}, \mathrm{t}_{\mathrm{R}}=4.8 \mathrm{~min}$, major; $\mathrm{t}_{\mathrm{R}}=6.6 \mathrm{~min}$, minor). Anal. Calcd for $\mathrm{C}_{19} \mathrm{H}_{20} \mathrm{BrNO}_{4}$ : C, 56.17; H, 4.96; N, 3.45. Found: C, 56.16; H, $4.89 ; \mathrm{N}, 3.43$. 


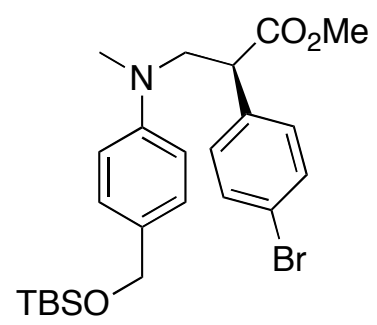

7f: Procedure A: pale yellow oil, $\mathbf{R}_{\mathrm{f}} 0.28$ (10:1 pentane/ether); [a] ${ }_{\mathrm{D}}^{25} \square 73.0$ (c 7.50, $\mathrm{CHCl}_{3}$ ); FTIR (film) 2952, 2928, 2855, 1736, 1615, 1522, 1488, 1256, 1164, $1074 \mathrm{~cm}^{-1}$; ${ }^{1} \mathrm{H}$ NMR (500 MHz, $\left.\mathrm{CDCl}_{3}\right) \square 7.51(\mathrm{~d}, J=8.4 \mathrm{~Hz}, 2 \mathrm{H}), 7.27(\mathrm{~d}, J=8.5 \mathrm{~Hz}, 2 \mathrm{H}), 7.25(\mathrm{~d}$, $J=8.4 \mathrm{~Hz}, 2 \mathrm{H}), 6.71(\mathrm{~d}, J=8.5 \mathrm{~Hz}, 2 \mathrm{H}), 4.71(\mathrm{~s}, 2 \mathrm{H}), 4.13(\mathrm{dd}, J=14.4,8.4 \mathrm{~Hz}, 1 \mathrm{H})$, $4.04(\mathrm{dd}, J=8.4,5.8 \mathrm{~Hz}, 1 \mathrm{H}), 3.69$ (s, 3H), $3.61(\mathrm{dd}, J=14.4,5.8 \mathrm{~Hz}, 1 \mathrm{H}), 2.84(\mathrm{~s}, 3 \mathrm{H})$, 1.00 (s, 9H), 0.15 (s, 6H); ${ }^{13} \mathrm{C}$ NMR (125 MHz, $\left.\mathrm{CDCl}_{3}\right) \square$ 173.0, 147.3, 136.0, 131.8, $129.8,129.6,127.7,121.6,112.0,64.9,56.7,52.2,48.8,39.4,26.0,18.4,-5.2$; LC-MS (ESI) $\mathrm{m} / z$ (relative intensity) $492\left([\mathrm{M}+\mathrm{H}]^{+}, 100\right)$; HPLC analysis: $61 \%$ ee (Chiralcel OJ, $3.0 \% i-\mathrm{PrOH}$ in hexane, $1.0 \mathrm{~mL} / \mathrm{min}, \mathrm{C}=254 \mathrm{~nm}, \mathrm{t}_{\mathrm{R}}=9.9$ min, major; $\mathrm{t}_{\mathrm{R}}=15.5 \mathrm{~min}$, minor). Anal. Calcd for $\mathrm{C}_{24} \mathrm{H}_{34} \mathrm{BrNO}_{3} \mathrm{Si}$ : C, 58.53; H, 6.96; N, 2.84. Found: C, 58.49; H, $6.90 ; \mathrm{N}, 2.85$.

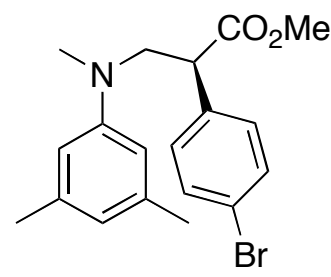

9: Procedure A: pale yellow oil, $\mathrm{R}_{\mathrm{f}} 0.58$ (5:1 pentane/ether); [a] ${ }_{\mathrm{D}}^{25} \square 86.8$ (c 6.30, $\mathrm{CHCl}_{3}$ ); FTIR (film) 2949, 2916, 1735, 1599, 1488, 1435, 1356, 1342, 1205, 1163, $1011 \mathrm{~cm}^{-1} ;{ }^{1} \mathrm{H}$ NMR (500 MHz, $\left.\mathrm{CDCl}_{3}\right) \square 7.45(\mathrm{~d}, J=8.4 \mathrm{~Hz}, 2 \mathrm{H}), 7.19(\mathrm{~d}, J=8.4 \mathrm{~Hz}, 2 \mathrm{H}), 6.41$ (s, 1H), $6.30(\mathrm{~s}, 2 \mathrm{H}), 4.04(\mathrm{dd}, J=14.2,8.0 \mathrm{~Hz}, 1 \mathrm{H}), 3.98$ (dd, $J=8.0,5.5 \mathrm{~Hz}, 1 \mathrm{H}), 3.64$ (s, $3 \mathrm{H}), 3.54(\mathrm{dd}, J=14.2,5.5 \mathrm{~Hz}, 1 \mathrm{H}), 2.78(\mathrm{~s}, 3 \mathrm{H}), 2.27$ (s, 3H); ${ }^{13} \mathrm{C}$ NMR $(125 \mathrm{MHz}$, $\left.\mathrm{CDCl}_{3}\right) \square 173.0,148.3,138.7,136.1,131.8,129.7,121.6,119.0,110.2,56.6,52.2,49.0$, 39.4, 21.8 (2C); LC-MS (ESI) m/z (relative intensity) 277 (18), 294 (12), 362 (12), 376 $\left([\mathrm{M}+\mathrm{H}]^{+}, 100\right)$; HPLC analysis: $64 \%$ ee (Chiralcel OJ, $3.0 \%$-PrOH in hexane, 1.0 $\mathrm{mL} / \mathrm{min}, \quad \square=254 \mathrm{~nm}, \mathrm{t}_{\mathrm{R}}=10.5$ min, minor; $\mathrm{t}_{\mathrm{R}}=12.6$ min, major). Anal. Calcd for $\mathrm{C}_{19} \mathrm{H}_{22} \mathrm{BrNO}_{2}$ : C, 60.65; H, 5.89; N, 3.72. Found: C, 60.91; H, 5.91; N, 3.64. 


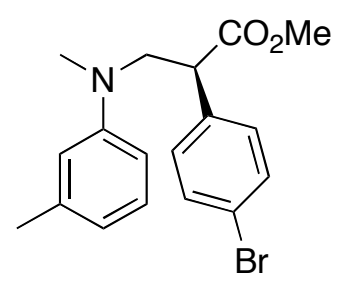

11: Procedure A: colorless oil, $\mathrm{R}_{\mathrm{f}} 0.38$ (10:1 pentane/ether); [a] ${ }_{\mathrm{D}}^{25} \square 80.7$ (c 1.20, $\mathrm{CHCl}_{3}$ ); FTIR (film) 2949, 2917, 1735, 1602, 1498, 1488, 1203, 1163, $1011 \mathrm{~cm}^{-1} ;{ }^{1} \mathrm{H}$ NMR (500 $\left.\mathrm{MHz}, \mathrm{CDCl}_{3}\right) \square 7.46(\mathrm{~d}, J=8.4 \mathrm{~Hz}, 2 \mathrm{H}), 7.20(\mathrm{~d}, J=8.4 \mathrm{~Hz}, 2 \mathrm{H}), 7.14(\mathrm{t}, J=7.9 \mathrm{~Hz}$, $1 \mathrm{H}), 6.57(\mathrm{~d}, J=7.6 \mathrm{~Hz}, 1 \mathrm{H}), 6.51-6.46(\mathrm{~m}, 2 \mathrm{H}), 4.06(\mathrm{dd}, J=14.6,8.0 \mathrm{~Hz}, 1 \mathrm{H}), 3.98$ (dd, $J=8.0,6.0 \mathrm{~Hz}, 1 \mathrm{H}), 3.65(\mathrm{~s}, 3 \mathrm{H}), 3.56(\mathrm{dd}, J=14.6,6.0 \mathrm{~Hz}, 1 \mathrm{H}), 2.79(\mathrm{~s}, 3 \mathrm{H}), 2.32$ (s, 3H); ${ }^{13} \mathrm{C}$ NMR (125 MHz, $\left.\mathrm{CDCl}_{3}\right) \square 173.1,148.3,139.0,136.1,131.9,129.8,129.1$, 121.6, 117.7, 113.0, 109.4, 56.6, 52.3, 49.0, 39.4, 21.9; LC-MS (ESI) $\mathrm{m} / \mathrm{z}$ (relative intensity) $362\left([\mathrm{M}+\mathrm{H}]^{+}, 100\right)$; HPLC analysis: $64 \%$ ee (Chiralcel OJ, $10.0 \% i-\mathrm{PrOH}$ in hexane, $1.0 \mathrm{~mL} / \mathrm{min}, \square=254 \mathrm{~nm}, \mathrm{t}_{\mathrm{R}}=11.6 \mathrm{~min}$, minor; $\mathrm{t}_{\mathrm{R}}=25.0 \mathrm{~min}$, major). Anal. Calcd for $\mathrm{C}_{18} \mathrm{H}_{20} \mathrm{BrNO}_{2}$ : C, 59.68; H, 5.56; N, 3.87. Found: C, 59.41; H, 5.60; N, 3.72.

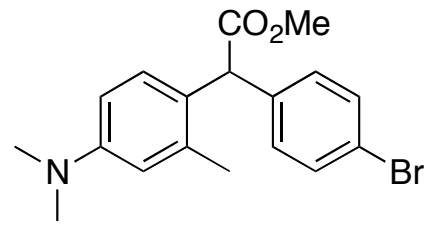

12: Procedure A: white solid, mp $77-78{ }^{\circ} \mathrm{C} ; \mathrm{R}_{\mathrm{f}} 0.15$ (10:1 pentane/ether); FTIR $\left(\mathrm{CDCl}_{3}\right)$ 2948, 2800, 1737, 1611, 1511, 1487, 1355, 1158, $1010 \mathrm{~cm}^{-1} ;{ }^{1} \mathrm{H}$ NMR (500 MHz, $\mathrm{CDCl}_{3}$ ) $77.40(\mathrm{~d}, J=8.2 \mathrm{~Hz}, 2 \mathrm{H}), 7.10(\mathrm{~d}, J=8.2 \mathrm{~Hz}, 2 \mathrm{H}), 7.06(\mathrm{~d}, J=8.2 \mathrm{~Hz}, 1 \mathrm{H}), 6.57-6.53$ (m, 2H), 5.07 (s, 1H), 3.72 (s, 3H), 2.91 (s, 6H), 2.21 (s, 3H); ${ }^{13} \mathrm{C}$ NMR (125 MHz, $\left.\mathrm{CDCl}_{3}\right) \square$ 173.3, 149.7, 137.8, 136.9, 131.4, 130.5, 128.6, 124.2, 120.9, 114.6, 110.2, 52.25, 52.22, 40.4, 20.2; LC-MS (ESI) $\mathrm{m} / z$ (relative intensity) 362 ([M+H] $]^{+}, 100$ ); Anal. Calcd for $\mathrm{C}_{18} \mathrm{H}_{20} \mathrm{BrNO}_{2}$ : C, 59.68; H, 5.56; N, 3.87. Found: C, 59.43; H, 5.48; N, 3.87 .

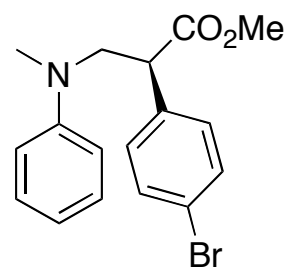


14: Procedure A: colorless oil, $\mathrm{R}_{\mathrm{f}} 0.38$ (10:1 pentane/ether); [a] ${ }_{\mathrm{D}}^{25} \square 64.4$ (c 0.50 , $\mathrm{CDCl}_{3}$ ); FTIR (film) 2949, 1735, 1600, 1506, 1488, 1164, $1011 \mathrm{~cm}^{-1} ;{ }^{1} \mathrm{H}$ NMR (500 $\left.\mathrm{MHz} \mathrm{CDCl}_{3}\right) \square 7.46(\mathrm{~d}, J=8.4 \mathrm{~Hz}, 2 \mathrm{H}), 7.25$ (pseudo t, $\left.J=8.1 \mathrm{~Hz}, 2 \mathrm{H}\right), 7.20$ (d, $J=8.4$ $\mathrm{Hz}, 2 \mathrm{H}), 6.74$ (br t, $J=7.7 \mathrm{~Hz}, 1 \mathrm{H}), 6.68$ (d, $J=8.2 \mathrm{~Hz}, 2 \mathrm{H}), 4.09$ (dd, $J=14.6,8.2 \mathrm{~Hz}$, 1H), 3.99 (dd, $J=8.2,6.0 \mathrm{~Hz}, 1 \mathrm{H}), 3.65$ (s, 3H), 3.57 (dd, $J=14.6,6.0 \mathrm{~Hz}, 1 \mathrm{H}), 2.80$ (s, $3 \mathrm{H}) ;{ }^{13} \mathrm{C}$ NMR $\left(125 \mathrm{MHz}, \mathrm{CDCl}_{3}\right) \square 173.0,148.2$, 136.0, 131.9, 129.8, 129.3, 121.7, 116.8, 112.2, 56.6, 52.3, 48.9, 39.3; MS (EI) $\mathrm{m} / \mathrm{z}$ (relative intensity) 120.0 (100), 347.1 $\left(\mathrm{M}^{+}, 2\right)$; HRMS (EI) $m / z$ Calcd for $\left[\mathrm{C}_{17} \mathrm{H}_{18} \mathrm{BrNO}_{2}\right]^{+}:$347.0515. Found: 347.05194. HPLC analysis: $48 \%$ ee (Chiralcel OJ, $10.0 \% \mathrm{i}$-PrOH in hexane, $1.0 \mathrm{~mL} / \mathrm{min}, \square=254 \mathrm{~nm}, \mathrm{t}_{\mathrm{R}}=$ 23.1 min, minor; $t_{R}=35.3$ min, major).

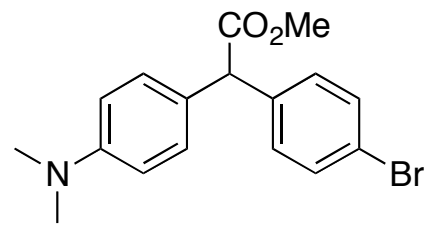

15: Procedure A: colorless oil: $\mathrm{R}_{\mathrm{f}} 0.25$ (5:1 pentane/ether); FTIR (film) 2948, 2888, 2802, 1736, 1613, 1522, 1488, 1351, 1194, 1154, $1010 \mathrm{~cm}^{-1} ;{ }^{1} \mathrm{H}$ NMR (400 MHz, $\left.\mathrm{CDCl}_{3}\right)$ $7.40(\mathrm{~d}, J=8.4 \mathrm{~Hz}, 2 \mathrm{H}), 7.16(\mathrm{~d}, J=8.4 \mathrm{~Hz}, 2 \mathrm{H}), 7.12(\mathrm{~d}, J=8.8 \mathrm{~Hz}, 2 \mathrm{H}), 6.66(\mathrm{~d}, J=$ $8.8 \mathrm{~Hz}, 2 \mathrm{H}), 4.87(\mathrm{~s}, 1 \mathrm{H}), 3.70(\mathrm{~s}, 3 \mathrm{H}), 2.90(\mathrm{~s}, 6 \mathrm{H}) ;{ }^{13} \mathrm{C}$ NMR $\left(75 \mathrm{MHz}, \mathrm{CDCl}_{3}\right) \square$ 173.0, 149.7, 138.4, 131.4, 130.2, 129.0, 125.6, 121.0, 112.5, 55.4, 52.2, 40.4; LC-MS (ESI) $\mathrm{m} / \mathrm{z}$ (relative intensity) $348\left([\mathrm{M}+\mathrm{H}]^{+}, 100\right)$; Anal. Calcd for $\mathrm{C}_{17} \mathrm{H}_{18} \mathrm{BrNO}_{2}$ : C, 58.63; H, 5.21; N, 4.02. Found: C, 58.64; H, 5.19; N, 4.02.

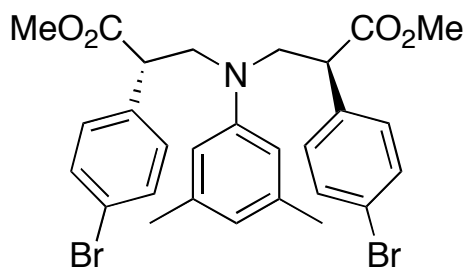

18: Procedure B: white solid: $\operatorname{mp} 113-114{ }^{\circ} \mathrm{C} ; \mathrm{R}_{\mathrm{f}} 0.38$ (5:1 pentane/ether); [a] $]_{\mathrm{D}}^{25} \square 123.2$ (c 4.00, $\left.\mathrm{CHCl}_{3}\right)$; FTIR $\left(\mathrm{CHCl}_{3}\right)$ 2999, 2917, 1735, 1598, 1487, 1435, 1407, 1353, 1261, 1203, 1166, 1073, $1011 \mathrm{~cm}^{-1}$; ${ }^{1} \mathrm{H}$ NMR (500 MHz, $\left.\mathrm{CDCl}_{3}\right) \square 7.46(\mathrm{~d}, J=8.2 \mathrm{~Hz}, 4 \mathrm{H})$, $7.15(\mathrm{~d}, J=8.2 \mathrm{~Hz}, 4 \mathrm{H}), 6.46(\mathrm{~s}, 1 \mathrm{H}), 6.28(\mathrm{~s}, 2 \mathrm{H}), 4.04(\mathrm{dd}, J=14.6,8.8 \mathrm{~Hz}, 2 \mathrm{H}), 3.93$ $(\mathrm{dd}, J=8.8,4.9 \mathrm{~Hz}, 2 \mathrm{H}), 3.60(\mathrm{~s}, 6 \mathrm{H}), 3.40(\mathrm{dd}, J=14.6,4.9 \mathrm{~Hz}, 2 \mathrm{H}), 2.28(\mathrm{~s}, 6 \mathrm{H}) ;{ }^{13} \mathrm{C}$ 
NMR (125 MHz, $\left.\mathrm{CDCl}_{3}\right) \square 172.9,145.8,139.0,136.0,131.9,129.6,121.6,119.7$, 111.7, 55.2, 52.2, 48.6, 21.8; LC-MS (ESI) $\mathrm{m} / z$ (relative intensity) $602\left([\mathrm{M}+\mathrm{H}]^{+}, 52\right)$; HPLC analysis: 95\% ee $((R, R)$-Whelk-O 1, 2.0\% $i$-PrOH in hexane, $1.0 \mathrm{~mL} / \mathrm{min}, \square=$ $254 \mathrm{~nm}, \mathrm{t}_{\mathrm{R}}=14.0 \mathrm{~min}$, major; $\mathrm{t}_{\mathrm{R}}=17.7 \mathrm{~min}$, minor). Anal. Calcd for $\mathrm{C}_{28} \mathrm{H}_{29} \mathrm{Br}_{2} \mathrm{NO}_{4}: \mathrm{C}$, 55.74; H, 4.84; N, 2.32. Found: C, 55.64; H, 4.85; N, 2.38.

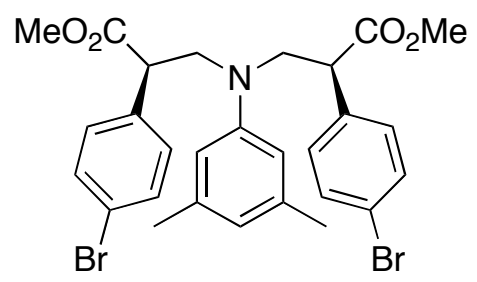

19: Procedure B: semi-solid; $\mathrm{R}_{\mathrm{f}} 0.25$ (5:1 pentane/ether); FTIR $\left(\mathrm{CDCl}_{3}\right)$ 2950, 2918, $1733,1598,1488,1435,1353,1207,1163,1073,1011 \mathrm{~cm}^{-1} ;{ }^{1} \mathrm{H}$ NMR $\left(500 \mathrm{MHz}, \mathrm{CDCl}_{3}\right)$ $\square 7.43(\mathrm{~d}, J=8.2 \mathrm{~Hz}, 4 \mathrm{H}), 7.08(\mathrm{~d}, J=8.2 \mathrm{~Hz}, 4 \mathrm{H}), 6.46(\mathrm{~s}, 1 \mathrm{H}), 6.26$ (s, 2H), 3.89 (dd, $J$ $=7.8,6.3 \mathrm{~Hz}, 2 \mathrm{H}), 3.78(\mathrm{dd}, J=14.8,7.8 \mathrm{~Hz}, 2 \mathrm{H}), 3.62(\mathrm{~s}, 6 \mathrm{H}), 3.42(\mathrm{dd}, J=14.8,6.3$ $\mathrm{Hz}, 2 \mathrm{H}), 2.78$ (s, 6H); ${ }^{13} \mathrm{C}$ NMR (125 MHz, $\left.\mathrm{CDCl}_{3}\right) \square 172.9,145.9,139.1,136.0,131.9$, 129.8, 121.6, 119.7, 111.6, 55.0, 52.2, 48.5, 21.9; LC-MS (ESI) $\mathrm{m} / z$ (relative intensity) $602\left([\mathrm{M}+\mathrm{H}]^{+}, 51\right)$; Anal. Calcd for $\mathrm{C}_{28} \mathrm{H}_{29} \mathrm{Br}_{2} \mathrm{NO}_{4}: \mathrm{C}, 55.74 ; \mathrm{H}, 4.84 ; \mathrm{N}, 2.32$. Found: $\mathrm{C}$, $55.64 ; \mathrm{H}, 4.87 ; \mathrm{N}, 2.38$.

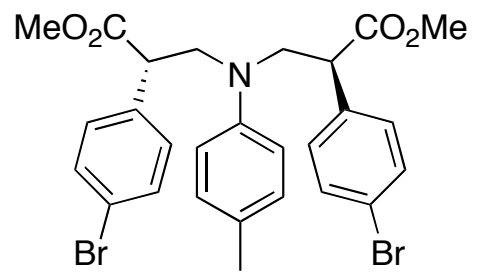

20a: Procedure B: white solid, $\mathrm{mp} 87-89^{\circ} \mathrm{C} ; \mathrm{R}_{\mathrm{f}} 0.25$ (5:1 pentane/ether); [a] ${ }_{\mathrm{D}}^{25} \square 120.8$ ( $c$ 3.60, $\left.\mathrm{CHCl}_{3}\right)$; FTIR $\left(\mathrm{CHCl}_{3}\right)$ 3024, 2950, 1735, 1618, 1518, 1488, 1202, 1166, 1073, 1011, 822, 804, $757 \mathrm{~cm}^{-1}$; ${ }^{1} \mathrm{H}$ NMR $\left(500 \mathrm{MHz}, \mathrm{CDCl}_{3}\right) \square 7.44(\mathrm{~d}, J=8.4 \mathrm{~Hz}, 4 \mathrm{H}), 7.14$ (d, $J=8.4 \mathrm{~Hz}, 4 \mathrm{H}), 7.09$ (d, $J=8.4 \mathrm{~Hz}, 2 \mathrm{H}), 6.62(\mathrm{~d}, J=8.4 \mathrm{~Hz}, 2 \mathrm{H}), 3.99(\mathrm{dd}, J=14.5,9.0$ $\mathrm{Hz}, 2 \mathrm{H}), 3.90$ (dd, $J=9.0,5.0 \mathrm{~Hz}, 2 \mathrm{H}), 3.59$ (s, 6H), 3.38 (dd, $J=14.5,5.0 \mathrm{~Hz}, 2 \mathrm{H}), 2.28$ (s, 3H); ${ }^{13} \mathrm{C}$ NMR (125 MHz, $\left.\mathrm{CDCl}_{3}\right) \square$ 172.9(C), 143.7(C), 135.9(C), 131.9(CH), 130.1(CH), 130.0(CH), 127.6(C), 121.6(C), $114.6(\mathrm{CH}), 55.6\left(\mathrm{CH}_{2}\right), 52.2\left(\mathrm{CH}_{3}\right), 48.6$ (CH), $20.3\left(\mathrm{CH}_{3}\right)$; LC-MS (ESI) $\mathrm{m} / z$ (relative intensity) $588\left([\mathrm{M}+\mathrm{H}]^{+}, 40\right), 610\left([\mathrm{M}+\mathrm{Na}]^{+}\right.$, 48); HPLC analysis: $91 \%$ ee $((R, R)$-Whelk-O 1, $5.0 \% i-\mathrm{PrOH}$ in hexane, $1.0 \mathrm{~mL} / \mathrm{min}$, 
$=254 \mathrm{~nm}, \mathrm{t}_{\mathrm{R}}=10.6$ min, major; $\mathrm{t}_{\mathrm{R}}=13.9$ min, minor). Anal. Calcd for $\mathrm{C}_{27} \mathrm{H}_{27} \mathrm{Br}_{2} \mathrm{NO}_{4}$ : C, 55.03; H, 4.62; N, 2.38. Found: C, 54.93; H, 4.64; N, 2.34.

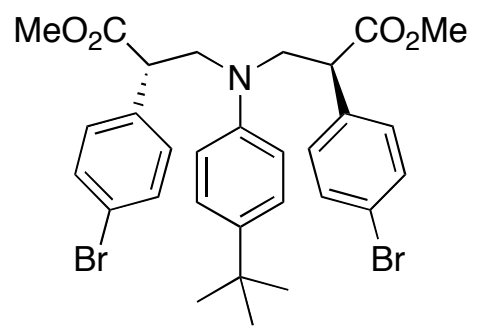

20b: Procedure B: white solid, mp $118-121{ }^{\circ} \mathrm{C} ; \mathrm{R}_{\mathrm{f}} 0.38$ (5:1 pentane/ether); [a] ${ }_{\mathrm{D}}^{25} \square 119.0$ (c 7.40, $\left.\mathrm{CHCl}_{3}\right)$; FTIR $\left(\mathrm{CHCl}_{3}\right)$ 2952, 1735, 1611, 1518, 1488, 1202, 1167, 1074, 1011 $\mathrm{cm}^{-1}$; ${ }^{1} \mathrm{H}$ NMR $\left(500 \mathrm{MHz}, \mathrm{CDCl}_{3}\right) \square 7.45(\mathrm{~d}, J=8.2 \mathrm{~Hz}, 4 \mathrm{H}), 7.29(\mathrm{~d}, J=8.8 \mathrm{~Hz}, 2 \mathrm{H})$, $7.16(\mathrm{~d}, J=8.2 \mathrm{~Hz}, 4 \mathrm{H}), 6.63(\mathrm{~d}, J=8.8 \mathrm{~Hz}, 2 \mathrm{H}), 4.05(\mathrm{dd}, J=14.6,9.1 \mathrm{~Hz}, 2 \mathrm{H}), 3.93$ (dd, $J=9.1,4.9 \mathrm{~Hz}, 2 \mathrm{H}), 3.59(\mathrm{~s}, 6 \mathrm{H}), 3.40(\mathrm{dd}, J=14.6,4.9 \mathrm{~Hz}, 2 \mathrm{H}), 1.31(\mathrm{~s}, 9 \mathrm{H}) ;{ }^{13} \mathrm{C}$ NMR (125 MHz, $\left.\mathrm{CDCl}_{3}\right) \square 172.9,143.6,140.5,135.9,131.9,129.6,126.3,121.6,113.6$, 55.3, 52.1 , 48.6, 33,7, 31.4; LC-MS (ESI) $\mathrm{m} / z$ (relative intensity) 402 (5), $630\left([\mathrm{M}+\mathrm{H}]^{+}\right.$, 13), $652\left([\mathrm{M}+\mathrm{Na}]^{+}, 46\right)$; HPLC analysis: $91 \%$ ee $((R, R)$-Whelk-O $1,3.0 \% i$-PrOH in hexane, $1.0 \mathrm{~mL} / \mathrm{min}, \square=254 \mathrm{~nm}, \mathrm{t}_{\mathrm{R}}=11.4 \mathrm{~min}$, major; $\mathrm{t}_{\mathrm{R}}=14.3 \mathrm{~min}$, minor). Anal. Calcd for $\mathrm{C}_{30} \mathrm{H}_{33} \mathrm{Br}_{2} \mathrm{NO}_{4}$ : C, 57.07; H, 5.27; N, 2.22. Found: C, 57.13; H, 5.27; N, 2.28.

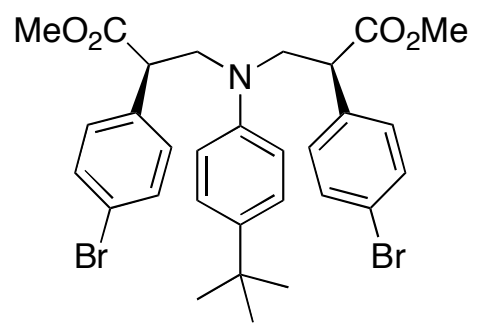

20b (meso): Procedure B: white solid, $\mathrm{mp} 108-111^{\circ} \mathrm{C} ; \mathrm{R}_{\mathrm{f}} 0.22$ (5:1 pentane/ether); FTIR $\left(\mathrm{CHCl}_{3}\right)$ 2952, 1735, 1610, 1518, 1488, 1199, 1165, 1073, $1011 \mathrm{~cm}^{-1} ;{ }^{1} \mathrm{H}$ NMR (500 $\left.\mathrm{MHz}, \mathrm{CDCl}_{3}\right) \square 7.42(\mathrm{~d}, J=8.2 \mathrm{~Hz}, 4 \mathrm{H}), 7.28(\mathrm{~d}, J=8.5 \mathrm{~Hz}, 2 \mathrm{H}), 7.10(\mathrm{~d}, J=8.2 \mathrm{~Hz}$, $4 \mathrm{H}), 6.64(\mathrm{~d}, J=8.5 \mathrm{~Hz}, 2 \mathrm{H}), 3.91-3.86(\mathrm{~m}, 2 \mathrm{H}), 3.81(\mathrm{dd}, J=14.5,7.8 \mathrm{~Hz}, 2 \mathrm{H}), 3.60(\mathrm{~s}$, $6 \mathrm{H}), 3.42(\mathrm{dd}, J=14.5,6.0 \mathrm{~Hz}, 2 \mathrm{H}), 1.31(\mathrm{~s}, 9 \mathrm{H}) ;{ }^{13} \mathrm{C} \mathrm{NMR}\left(125 \mathrm{MHz}, \mathrm{CDCl}_{3}\right) \square 173.1$, 143.5 (weak), 140.6 (weak), 136.2, 132.1, 130.1, 126.6, 121.9, 114.0, 55.5, 52.5, 48.8, 34,1, 31.7; LC-MS (ESI) $\mathrm{m} / \mathrm{z}$ (relative intensity) $402(6), 630\left([\mathrm{M}+\mathrm{H}]^{+}, 10\right), 652$ 
([M+Na $]^{+}$, 51); Anal. Calcd for $\mathrm{C}_{30} \mathrm{H}_{33} \mathrm{Br}_{2} \mathrm{NO}_{4}: \mathrm{C}, 57.07 ; \mathrm{H}, 5.27 ; \mathrm{N}, 2.22$. Found: $\mathrm{C}$, 56.79; H, 5.22; N, 2.36.

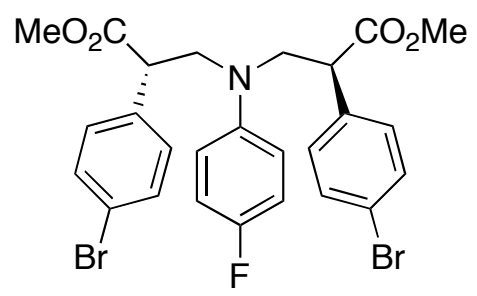

20c: Procedure B: pale yellow oil; $R_{\mathrm{f}} 0.25$ (5:1 pentane/ether); [a] $]_{\mathrm{D}}^{25} \square 97.2$ (c 5.00, $\mathrm{CHCl}_{3}$ ); FTIR (film) 2951, 1734, 1510, 1488, 1231, 1204, 1166, 1073, $1011 \mathrm{~cm}^{-1} ;{ }^{1} \mathrm{H}$ NMR (500 MHz, $\left.\mathrm{CDCl}_{3}\right) \square 7.48(\mathrm{~d}, J=8.5 \mathrm{~Hz}, 4 \mathrm{H}), 7.16(\mathrm{~d}, J=8.5 \mathrm{~Hz}, 4 \mathrm{H}), 7.01$ (dd, $J$ $=8.8,8.6 \mathrm{~Hz}, 2 \mathrm{H}), 6.70(\mathrm{dd}, J=8.8,4.3 \mathrm{~Hz}, 2 \mathrm{H}), 3.99(\mathrm{dd}, J=14.5,8.9 \mathrm{~Hz}, 2 \mathrm{H}), 3.88$ $(\mathrm{dd}, J=8.9,5.2 \mathrm{~Hz}, 2 \mathrm{H}), 3.64(\mathrm{~s}, 6 \mathrm{H}), 3.39(\mathrm{dd}, J=14.5,5.2 \mathrm{~Hz}, 2 \mathrm{H}) ;{ }^{13} \mathrm{C}$ NMR $(125$ $\left.\mathrm{MHz} \mathrm{CDCl}_{3}\right) \square 172.8,156.4(\mathrm{~d}, J=238.5 \mathrm{~Hz}), 142.8$ (d, $\left.J=1.6 \mathrm{~Hz}\right), 135.6,131.9,129.6$, 121.7, $116.6(\mathrm{~d}, J=7.3 \mathrm{~Hz}), 116.0(\mathrm{~d}, J=22.0 \mathrm{~Hz}), 56.1,52.2,48.7$; MS (EI) $\mathrm{m} / z$ (relative intensity) 198.9 (29), 364.1 (100), $591.2\left(\mathrm{M}^{+}, 2\right)$; HRMS (EI) $\mathrm{m} / z$ Calcd for $\left[\mathrm{C}_{26} \mathrm{H}_{25} \mathrm{Br}_{2} \mathrm{FNO}_{4}\right]^{+}:$591.0051. Found: 591.00317. HPLC analysis: $89 \%$ ee $((R, R)-$ Whelk$\mathrm{O} 1,5.0 \% i-\mathrm{PrOH}$ in hexane, $1.0 \mathrm{~mL} / \mathrm{min}, \square=254 \mathrm{~nm}, \mathrm{t}_{\mathrm{R}}=12.0 \mathrm{~min}$, major; $\mathrm{t}_{\mathrm{R}}=16.0$ min, minor).

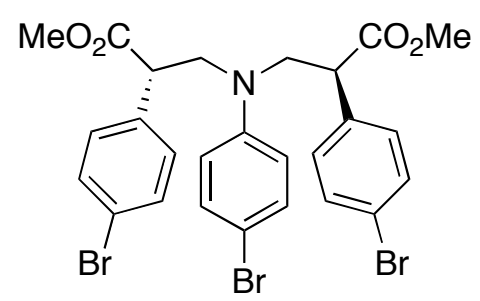

20d: Procedure B: white solid, $m p 120-122{ }^{\circ} \mathrm{C} ; \mathrm{R}_{\mathrm{f}} 0.28$ (5:1 pentane/ether); [a] ${ }_{\mathrm{D}}^{25} \square 138.7$ (c 3.00, $\left.\mathrm{CHCl}_{3}\right)$; FTIR $\left(\mathrm{CHCl}_{3}\right)$ 2950, 1735, 1590, 1489, 1203, 1168, 1074, $1011 \mathrm{~cm}^{-1}$; ${ }^{1} \mathrm{H}$ NMR (500 MHz, CDCl $) \square 7.46(\mathrm{~d}, J=8.4 \mathrm{~Hz}, 4 \mathrm{H}), 7.35(\mathrm{~d}, J=8.8 \mathrm{~Hz}, 2 \mathrm{H}), 7.13(\mathrm{~d}, J=$ $8.4 \mathrm{~Hz}, 4 \mathrm{H}), 6.55(\mathrm{~d}, J=8.8 \mathrm{~Hz}, 2 \mathrm{H}), 4.01(\mathrm{dd}, J=15.0,8.9 \mathrm{~Hz}, 2 \mathrm{H}), 3.88(\mathrm{dd}, J=8.9$, $5.5 \mathrm{~Hz}, 2 \mathrm{H}), 3.60(\mathrm{~s}, 6 \mathrm{H}), 3.36(\mathrm{dd}, J=15.0,5.5 \mathrm{~Hz}, 2 \mathrm{H}) ;{ }^{13} \mathrm{C} \mathrm{NMR}\left(125 \mathrm{MHz}, \mathrm{CDCl}_{3}\right) \square$ 172.6, 144.8, 135.4, 132.3, 132.0, 129.6, 121.8, 115.4, 110.0, 55.2, 52.3, 48.3; LC-MS (ESI) $m / z$ (relative intensity) $652\left([\mathrm{M}+\mathrm{H}]^{+}, 16\right), 674\left([\mathrm{M}+\mathrm{Na}]^{+}, 26\right)$; HPLC analysis: 90\% ee $\left((R, R)\right.$-Whelk-O 1, 5.0\% $i$-PrOH in hexane, $1.0 \mathrm{~mL} / \mathrm{min}, \square=254 \mathrm{~nm}, \mathrm{t}_{\mathrm{R}}=14.3 \mathrm{~min}$, 
major; $\mathrm{t}_{\mathrm{R}}=17.8$ min, minor). Anal. Calcd for $\mathrm{C}_{26} \mathrm{H}_{24} \mathrm{Br}_{3} \mathrm{NO}_{4}: \mathrm{C}, 47.74 ; \mathrm{H}, 3.70 ; \mathrm{N}, 2.14$. Found: C, 47.44; H, 3.55; N, 2.08 .

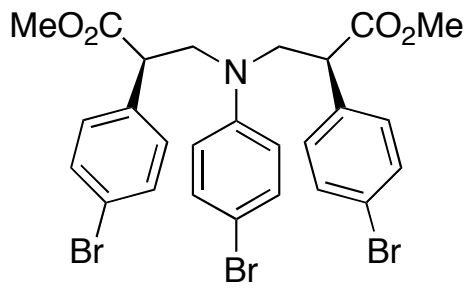

20d (meso): Procedure B: white solid, $105-110{ }^{\circ} \mathrm{C} ; \mathrm{R}_{\mathrm{f}} 0.18$ (5:1 pentane/ether); FTIR $\left(\mathrm{CHCl}_{3}\right)$ 2950, 1733, 1590, 1201, 1166, 1073, $1011 \mathrm{~cm}^{-1} ;{ }^{1} \mathrm{H}$ NMR $\left(500 \mathrm{MHz}, \mathrm{CDCl}_{3}\right) \square$ $7.43(\mathrm{~d}, J=8.5 \mathrm{~Hz}, 4 \mathrm{H}), 7.36(\mathrm{~d}, J=8.8 \mathrm{~Hz}, 2 \mathrm{H}), 7.07(\mathrm{~d}, J=8.5 \mathrm{~Hz}, 4 \mathrm{H}), 6.58(\mathrm{~d}, J=$ $8.8 \mathrm{~Hz}, 2 \mathrm{H}), 3.85$ (dd, $J=8.0,5.8 \mathrm{~Hz}, 2 \mathrm{H}), 3.80$ (dd, $J=14.3,8.0 \mathrm{~Hz}, 2 \mathrm{H}), 3.61(\mathrm{~s}, 6 \mathrm{H})$, $3.41(\mathrm{dd}, J=14.3,5.8 \mathrm{~Hz}, 2 \mathrm{H}) ;{ }^{13} \mathrm{C} \mathrm{NMR}\left(125 \mathrm{MHz}, \mathrm{CDCl}_{3}\right) \square 172.6,144.7,135.4$, $132.4,132.0,129.7,121.9,115.6,110.2$, 55.1, 52.4, 48.2; MS (FAB) $m / z$ (relative intensity) 136 (97), 154 (100), 424 (32), $652\left([\mathrm{M}+\mathrm{H}]^{+}, 6\right)$; HRMS (FAB) $m / z$ Calcd for $\left[\mathrm{C}_{26} \mathrm{H}_{25} \mathrm{Br}_{3} \mathrm{NO}_{4}\right]^{+}\left([\mathrm{M}+\mathrm{H}]^{+}\right):$651.9328. Found: 651.93159. Anal. Calcd for $\mathrm{C}_{26} \mathrm{H}_{24} \mathrm{Br}_{3} \mathrm{NO}_{4}$ : C, 47.74; H, 3.70; N, 2.14. Found: C, 47.64; H, 3.86; N, 2.07.

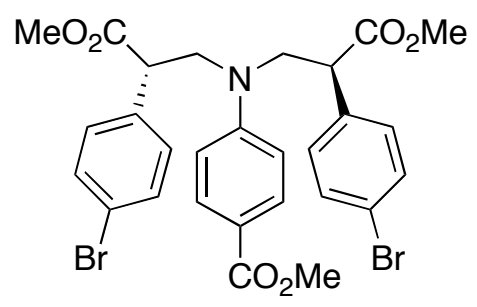

20e: Procedure $\mathbf{B}$ (run the reaction at $\left.50^{\circ} \mathrm{C}\right)$ : white solid, $147-148{ }^{\circ} \mathrm{C}\left(\mathrm{Et}_{2} \mathrm{O}\right) ; \mathrm{R}_{\mathrm{f}} 0.50(1: 1$ pentane/ether); [a] ${ }_{\mathrm{D}}^{25} \square 250.7$ ( $c 1.20, \mathrm{CHCl}_{3},>99 \%$ ee); FTIR $\left(\mathrm{CHCl}_{3}\right)$ 2950, 1734, 1708, 1606, 1520, 1488, 1435, 1285, 1202, 1185, $1170 \mathrm{~cm}^{-1} ;{ }^{1} \mathrm{H}$ NMR $\left(500 \mathrm{MHz}, \mathrm{CDCl}_{3}\right) \square$ $7.96(\mathrm{~d}, J=9.0 \mathrm{~Hz}, 2 \mathrm{H}), 7.48(\mathrm{~d}, J=8.2 \mathrm{~Hz}, 4 \mathrm{H}), 7.14(\mathrm{~d}, J=8.2 \mathrm{~Hz}, 4 \mathrm{H}), 6.62(\mathrm{~d}, J=$ $9.0 \mathrm{~Hz}, 2 \mathrm{H}), 4.11(\mathrm{dd}, J=15.0,8.8 \mathrm{~Hz}, 2 \mathrm{H}), 3.92(\mathrm{dd}, J=8.8,5.5 \mathrm{~Hz}, 2 \mathrm{H}), 3.88(\mathrm{~s}, 3 \mathrm{H})$, $3.61(\mathrm{~s}, 6 \mathrm{H}), 3.44(\mathrm{dd}, J=15.0,5.5 \mathrm{~Hz}, 2 \mathrm{H}) ;{ }^{13} \mathrm{C} \mathrm{NMR}\left(125 \mathrm{MHz}, \mathrm{CDCl}_{3}\right) \square 172.5,167.1$, 149.2, 135.3, 132.1, 131.7, 129.6, 122.0, 118.6, 111.8, 54.7, 52.4, 51.7, 48.3; MS (EI) $m / z$ (relative intensity) 132.0 (16), 199.0 (30), 404.0 (100), 600.0 (3), $631.0\left(\mathrm{M}^{+}, 2\right)$; HRMS (EI) $m / z$ Calcd for $\left[\mathrm{C}_{28} \mathrm{H}_{27} \mathrm{Br}_{2} \mathrm{NO}_{6}\right]^{+}:$631.0200. Found: 631.0137 . HPLC analysis: $85 \%$ ee (Chiralpak AD-RH, 5.0\% $i-\mathrm{PrOH}$ in hexane, $1.0 \mathrm{~mL} / \mathrm{min}, \square=254 \mathrm{~nm}, \mathrm{t}_{\mathrm{R}}=19.9 \mathrm{~min}$, 
minor; $\mathrm{t}_{\mathrm{R}}=23.0$ min, major); Anal. Calcd for $\mathrm{C}_{28} \mathrm{H}_{27} \mathrm{Br}_{2} \mathrm{NO}_{6}: \mathrm{C}, 53.10 ; \mathrm{H}, 4.30 ; \mathrm{N}, 2.21$. Found: C, 52.92; H, 4.25; N, 2.23.

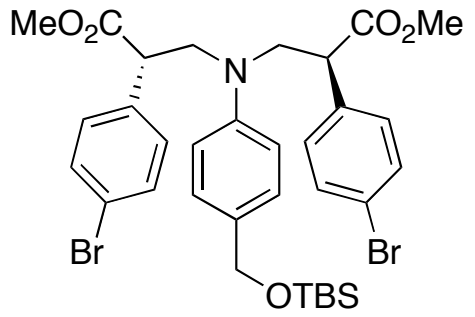

20f: Procedure B: pale yellow oil; $\mathrm{R}_{\mathrm{f}} 0.43$ (5:1 pentane/ether); [a] ${ }_{\mathrm{D}}^{25} \square 123.0$ (c 6.50, $\mathrm{CHCl}_{3}$ ); FTIR (film) 2951, 2928, 2855, 1736, 1615, 1519, 1488, 1359, 1258, 1202, 1167, 1074, $1011 \mathrm{~cm}^{-1}$; ${ }^{1} \mathrm{H}$ NMR $\left(500 \mathrm{MHz}, \mathrm{CDCl}_{3}\right) \square 7.45$ (d, $\left.J=8.4 \mathrm{~Hz}, 4 \mathrm{H}\right), 7.24$ (d, $J=8.5$ $\mathrm{Hz}, 2 \mathrm{H}), 7.14$ (d, $J=8.4 \mathrm{~Hz}, 4 \mathrm{H}), 6.66(\mathrm{~d}, J=8.5 \mathrm{~Hz}, 2 \mathrm{H}), 4.67$ (s, 2H), 4.02 (dd, $J=$ 14.6, $9.0 \mathrm{~Hz}, 2 \mathrm{H}), 3.92(\mathrm{dd}, J=9.0,5.2 \mathrm{~Hz}, 2 \mathrm{H}), 3.59$ (s, 6H), 3.40 (dd, $J=14.6,5.2 \mathrm{~Hz}$, 2H), 0.96 (s, 9H), 0.12 (s, 6H); ${ }^{13} \mathrm{C}$ NMR (125 MHz, $\left.\mathrm{CDCl}_{3}\right) \square$ 172.9, 144.9, 135.8, $131.9,130.7,130.0,127.9,121.7,113.8,64.7,55.4,52.2,48.4,26.0,18.5,-5.2$; MS (EI) $\mathrm{m} / z$ (relative intensity) 118.1 (19), 199.0 (14), 250.1 (10), 374.1 (7), 490.2 (100), 586.0 (5), 717.1 ( $\left.\mathrm{M}^{+}, 3\right)$; HRMS (EI) $\mathrm{m} / z$ Calcd for $\left[\mathrm{C}_{33} \mathrm{H}_{41}{ }^{81} \mathrm{Br}_{2} \mathrm{NO}_{5} \mathrm{Si}\right]^{+}:$721.1074. Found: 721.1073. HPLC analysis: $92 \%$ ee $((R, R)-$ Whelk-O $1,2.0 \% i$-PrOH in hexane, 1.0 $\mathrm{mL} / \mathrm{min}, \square=254 \mathrm{~nm}, \mathrm{t}_{\mathrm{R}}=13.6$ min, major; $\mathrm{t}_{\mathrm{R}}=17.0 \mathrm{~min}$, minor).

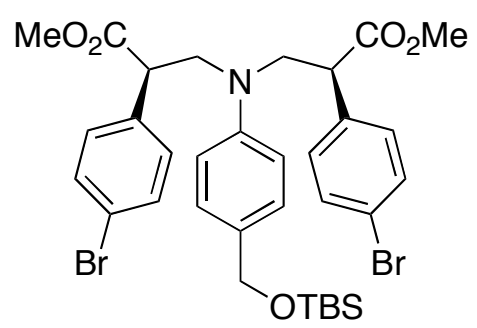

$20 f$ (meso): Procedure B: colorless oil; $\mathrm{R}_{\mathrm{f}} 0.38$ (5:1 pentane/ether); FTIR (film) 2951, 2928, 2855, 1735, 1615, 1519, 1488, 1257, 1165, 1074, $1011 \mathrm{~cm}^{-1} ;{ }^{1} \mathrm{H}$ NMR (500 MHz, $\left.\mathrm{CDCl}_{3}\right) \square 7.42(\mathrm{~d}, J=8.4 \mathrm{~Hz}, 4 \mathrm{H}), 7.23(\mathrm{~d}, J=8.5 \mathrm{~Hz}, 2 \mathrm{H}), 7.08(\mathrm{~d}, J=8.4 \mathrm{~Hz}, 4 \mathrm{H}), 6.64$ $(\mathrm{d}, J=8.5 \mathrm{~Hz}, 2 \mathrm{H}), 4.67(\mathrm{~s}, 2 \mathrm{H}), 3.87(\mathrm{dd}, J=8.0,6.1 \mathrm{~Hz}, 2 \mathrm{H}), 3.80$ (dd, $J=14.6,8.0$ $\mathrm{Hz}, 2 \mathrm{H}), 3.61(\mathrm{~s}, 6 \mathrm{H}), 3.43(\mathrm{dd}, J=14.6,6.1 \mathrm{~Hz}, 2 \mathrm{H}), 0.95(\mathrm{~s}, 9 \mathrm{H}), 0.12(\mathrm{~s}, 6 \mathrm{H}) ;{ }^{13} \mathrm{C}$ NMR (125 MHz, $\left.\mathrm{CDCl}_{3}\right) \square 172.8,144.9,135.8,131.8,130.7,129.8,127.9,121.6,113.7$, 64.8, 55.2, 52.2, 48.4, 26.0, 18.5, -5.2; LC-MS (ESI) $\mathrm{m} / \mathrm{z}$ (relative intensity) 492 (38), 718 
$\left([\mathrm{M}+\mathrm{H}]^{+}, 48\right)$; Anal. Calcd for $\mathrm{C}_{33} \mathrm{H}_{41} \mathrm{Br}_{2} \mathrm{NO}_{5} \mathrm{Si}$ : C, 55.08; H, 5.74; N, 1.95. Found: $\mathrm{C}$, $54.78 ; \mathrm{H}, 5.70 ; \mathrm{N}, 1.86$. 